\title{
OPEN The predictive value of soluble osteoclast-associated receptor for the prognosis of acute coronary syndrome
}

\author{
Rong Wang ${ }^{1}$, Jing Wang ${ }^{2}$, Ling Xie ${ }^{2}$, Hong-li Cai ${ }^{3}$, Yi Zhang $^{4}$ \& Oing Zhang ${ }^{3 凶}$
}

At present, prognostic biomarkers of acute coronary syndrome (ACS) are fewer. The aim of this study was to explore the predictive value of soluble osteoclast-associated receptor (sOSCAR) level for the major adverse cardiovascular events (MACE) occurring within 30 days after ACS. From January to August 2020, a total of 108 patients with ACS who were admitted to our hospital, were enrolled in this study. Of the 108 patients, 79 were men and 29 women. Patient-related data, including age, sex, body mass index, history of type 2 diabetes, history of hyperlipidemia and serum sOSCAR level, were collected. All patients were followed up for 30 days. Based on MACE occurrence, the 108 patients were divided into MACE group $(n=17)$ and non-MACE group $(n=91)$. The baseline data were compared between the two groups, MACE-independent risk factors were identified by multivariate regression analysis, and the predictive value of SOSCAR for MACE occurring within 30 days after CAS was analyzed using receiver operating characteristic $(R O C)$ curve. At the same time, according to the type of ACS, the 108 patients with ACS were divided into unstable angina (UA) group $(n=29)$, non ST-segment elevation myocardial infarction (USTEMI) group $(n=45)$ and ST-segment elevation myocardial infarction (STEMI) group $(n=34)$, and then the sOSCAR level and MACE incidence were observed in each group. The serum sOSCAR level was significantly lower in the MACE group $[130(100,183)]$ than in the non-MACE group $[301(220,370)](P=0.000)$. The area under ROC curve of sOSCAR level for MACE occurring within 30 days after CAS was 0.860 with $95 \% \mathrm{Cl} 0.782-0.919$, $P<0.001$. Multivariate regression analysis indicated that the sOSCAR level was an independent risk factor for the MACE occurring within 30 days after CAS (OR $0.26,95 \% \mathrm{Cl} 0.087-0.777, P=0.04)$. The MACE incidence (0\%) was the lowest but the sOSCAR level was the highest in the UA group, while in the STEMI group, the MACE incidence (23.53\%) was the higest but the sOSCAR level was the lowest among the UA, STEMI and NSTEMI groups. Serum sOSCAR level may be used as a predictor of MACE occurring within the short-term after ACS. The higher the sOSCAR level, the lower the MACE incidence.

Acute coronary syndrome (ACS) is characterized by rupture or invasion of coronary atherosclerotic plaques, thrombosis, myocardial ischemia, and it seriously threatens human life, health and safety ${ }^{1}$. At present, ACSprognostic biomarkers are fewer because the ACS-pathological mechanisms is not clear. Osteoclast-associated receptor (OSCAR), a costimulatory receptor, is involved in osteoclast differentiation. OSCAR has two forms including soluble and membranous ones ${ }^{2}$. In the endothelial cells of vascular wall, there are OSCAR expression which is regulated by oxidized low density lipoprotein (oxLDL), and is involved in the occurrence and progression of atherosclerosis ${ }^{3}$. Therefore, we speculate that OSCAR plays an important role in the occurrence of cardiovascular disease. However, it is not clear whether soluble osteoclast-associated receptor (sOSCAR) level plays a predictive role in ACS prognosis. The aim of this study was to explore the predictive value of sOSCAR level for short-term prognosis of ACS.

\footnotetext{
${ }^{1}$ The Department of Nephrology, Affiliated Hospital 2 of Nantong University, Nantong 226001, China. ${ }^{2}$ The Department of Cardiology, Affiliated Hospital 2 of Nantong University, Nantong 226001, China. ${ }^{3}$ The Department of General Practice, Affiliated Hospital 2 of Nantong University, No. 6, Hai'er Xiang North Road, Chongchuan District, Nantong 226001, China. ${ }^{4}$ The Department of Scientific Research, Affiliated Hospital 2 of Nantong University, Nantong 226001, China. ${ }^{\boxplus}$ email: zzhangqing32@sina.cn
} 


\section{Subjects and methods}

All study methods were approved by the Ethics Committee of the Second Affiliated Hospital of Nantong University (2019KS035), and were performed in accordance with relevant guidelines and regulations. All the subjects enrolled into the study gave written informed consent to participate.

Subjects and grouping. From January to August 2020, the patients who were admitted to our hospital for ACS, were collected. The inclusion criteria were the patients with ACS diagnosed according to clinical features, symptoms and coronary angiography ${ }^{4,5}$. The exclusion criteria were the patients with recent infections, osteoarthropathy, autoimmune diseases, malignant tumors, severe liver and kidney dysfunction, or chronic inflammatory diseases. Based on above inclusion and exclusion criteria, a total of 108 patients with ACS were enrolled in this study. Of the 108 patients, 79 were men and 29 women with a mean age of $66.94 \pm 11.08$ year. All patients were followed up for 30 days.

Based on the occurrence of major adverse cardiovascular events (MACE), the 108 patients were divided into MACE group $(n=17)$ and non-MACE group $(n=91)$.

According to the type of ACS, the 108 patients with ACS were divided into unstable angina (UA) group $(n=29)$, non ST-segment elevation myocardial infarction (USTEMI) group $(n=45)$ and ST-segment elevation myocardial infarction (STEMI) group $(\mathrm{n}=34)$.

Observation indices. The clinical data of all patients were recorded, including age, sex, history of smoking, history of hypertension, history of type 2 diabetes, history of hyperlipidemia, blood pressure and heart rate. In the morning of the next day after admission, fasting blood was taken from all patients for determining creatinine (Scr), troponin (TnI), total cholesterol (TC), high density lipoprotein (HDL), low density lipoprotein (LDL), triglyceride (TG), hypersensitive C-reactive protein (CRP), serum $\mathrm{N}$-terminal pro-brain natriuretic peptide (NT-proBNP), and all patients receiced the examination of left ventricular ejection fraction (LVEF). Above clinical data first uderwent single factor analysis, and then the factors with significant differences between the two groups in the single factor analysis further underwent multivariate Logistic regression analysis.

The sOSCAR level and MACE incidence were observed in the UA, USTEMI and STEMI groups, respectively.

Determination of sOSCAR level. In the morning of the next day after admission, $4 \mathrm{ml}$ of fasting blood was taken from all patients for determining sOSCAR. The fasting blood was centrifuged at $2500 \mathrm{r} / \mathrm{min}$ for $10 \mathrm{~min}$, and then the supernatant was used to determine the sOSCAR level according to the instructions of enzyme linked immunosorbent assay kit provided by BioTSZ Company (Hong Kong, China).

Follow-up. All patients were followed up by outpatient or telephone for 30 days after admission. The study endpoint was the occurrence of MACE. The MACE included death, heart failure, unstable angina and malignant arrhythmias. Within the 30 days, once the MACE occurred, the follow-up for the patient was stopped; otherwise the follow-up lasted 30 days.

Statistical analysis. The measurement data with normal distribution were expressed as mean \pm standard deviation $(\mathrm{x} \pm \mathrm{s}$ ), and the measurement data with non-normal distribution were expressed as P50 (P25-P75). The enumeration data were expressed as percentage or frequency. Independent sample $t$ test was used in the measurement data with normal distribution, $\chi^{2}$ test in the enumeration data, and Mann-Whitney $U$ test in the measurement data with non-normal distribution. The receiver operating characteristic (ROC) curve was drawn, and the area under the curve (AUC) was used to evaluate the predictive value of sOSCAR for the MACE occurring within 30 days after ACS. Bivariate Logistic regression was used to identify independent risk factors of MACE in ACS. Statistical analysis was performed using SPSS 23.0 and Med-Calc (version 11.2.1; MedCalc) softwares. Statistical significance was established at $P<0.05$.

\section{Results}

Comparisons of baseline data between the MACE group and non-MACE group. Age, Scr and NT-proBNP levels were significantly higher, but sOSCAR level and LVEF were significantly lower in the MACE group than in the non-MACE group (All $P<0.05$ ) (Table 1).

ROC curve of sOSCAR level for predicting MACE occurring within 30 days after ACS. The area under ROC curve of sOSCAR level for predicting MACE occurring within 30 days after CAS was 0.860 with $95 \%$ CI $0.782-0.919, P<0.001$, cut-off of $190 \mathrm{pg} / \mathrm{ml}$, specificity of $76.84 \%$ and sensitivity of $87.50 \%$ (Fig. 1 ).

Independent risk factors of MACE occurring within 30 days after ACS by multivariate Logistic regression analysis. The above 5 factors, including age, LVEF, sOSCAR, NT-proBNP and Scr, showed significant differences between the two groups, so the 5 factors, further underwent multivariate Logistic regression analysis. Multivariate Logistic regression analysis indicated that low serum sOSCAR (OR 0.26, 95\% CI 0.087$0.777, P=0.04$ ), high NT-proBNP (OR 6.13, 95\% CI $1.247-30.179, P=0.01$ ) and low LVEF value (OR $0.21,95 \%$ CI $0.072-0.613, P=0.03$ ) were independent risk factors of MACE occurring within 30 days after ACS (Table 2).

sOSCAR level in men and women. In this study, there were 79 men and 29 women. The sOSCAR level was $253.96 \pm 131.01 \mathrm{pg} / \mathrm{ml}$ in men and $274.55 \pm 101.08 \mathrm{pg} / \mathrm{ml}$ in women, and there was not significantly different between men and women in the sOSCAR level $(P=0.394)$. 


\begin{tabular}{|l|l|l|l|}
\hline & $\begin{array}{l}\text { MACE group } \\
(\mathbf{n}=\mathbf{1 7})\end{array}$ & $\begin{array}{l}\text { Non-MACE group } \\
(\mathbf{n = 9 1 )}\end{array}$ & P value \\
\hline Age (years) & $72.75 \pm 10.15$ & $63.72 \pm 12.66$ & 0.008 \\
\hline Gender (female) & $7(41.18)$ & $22(24.18)$ & 0.147 \\
\hline Type 2 diabetes & $3(17.65)$ & $20(21.98)$ & 0.487 \\
\hline Hypertension & $13(76.47)$ & $40(43.96)$ & 0.116 \\
\hline Hyperlipoidemia & $7(41.18)$ & $35(38.46)$ & 0.833 \\
\hline Smoking & $5(29.41)$ & $47(51.65)$ & 0.150 \\
\hline Stroke & $5(29.41)$ & $13(14.29)$ & 0.109 \\
\hline Heart rate $(\mathrm{beats} / \mathrm{min})$ & $79.87 \pm 15.96$ & $79.96 \pm 15.41$ & 0.983 \\
\hline Systolic pressure $(\mathrm{mmHg})$ & $129.68 \pm 20.73$ & $135.74 \pm 25.29$ & 0.366 \\
\hline Diastolic pressure $(\mathrm{mmHg})$ & $77.87 \pm 7.89$ & $81.07 \pm 18.96$ & 0.254 \\
\hline Triglyceride $(\mathrm{mmol} / \mathrm{L})$ & $1.62 \pm 0.75$ & $2.09 \pm 0.86$ & 0.311 \\
\hline Cholesterol $(\mathrm{mmol} / \mathrm{L})$ & $3.90 \pm 0.66$ & $4.47 \pm 1.19$ & 0.075 \\
\hline HDL $(\mathrm{mmol} / \mathrm{L})$ & $1.15 \pm 0.31$ & $1.15 \pm 0.25$ & 0.993 \\
\hline LDL $(\mathrm{mmol} / \mathrm{L})$ & $2.30 \pm 0.58$ & $2.84 \pm 1.10$ & 0.065 \\
\hline sOSCAR $(\mathrm{pg} / \mathrm{ml})$ & $130(100,183)$ & $301(220,370)$ & 0.000 \\
\hline Troponin $\mathrm{I}(\mu \mathrm{g} / \mathrm{L})$ & $2.53(0.72,7.17)$ & $2.39(0.01,11.71)$ & 0.625 \\
\hline hs-CRP & $14.51(4.90,50.18)$ & $7.75(2.35,21.33)$ & 0.207 \\
\hline NT-proBNP $(\mathrm{pg} / \mathrm{mL})$ & $5314(2231,9334)$ & $841(300,1715)$ & 0.013 \\
\hline Creatinine $(\mu \mathrm{mol} / \mathrm{L})$ & $87(70,115)$ & $72(61,91)$ & 0.032 \\
\hline LVEF $(\%)$ & $49.73 \pm 10.63$ & $61.34 \pm 8.01$ & 0.001 \\
\hline & & & \\
\hline
\end{tabular}

Table 1. Comparisons of baseline data between the MACE group and non-MACE group [n, $\%, x \pm s, P 50$ (P25-P75)]. MACE major adverse cardiovascular events; LVEF left ventricular ejection fraction; NT-proBNP $\mathrm{N}$-terminal pro-brain natriuretic peptide; $h s$-CRP hypersensitivity $\mathrm{C}$ reactive protein; $s O S C A R$ soluble osteoclast-associated receptor; HDL: high density lipoprotein; $L D L$ low density lipoprotein.

The sOSCAR level and MACE incidence in the UA, USTEMI and STEMI groups. According to the type of ACS, the 108 patients with ACS were divided into UA group $(n=29)$, USTEMI group $(n=45)$ and STEMI group $(\mathrm{n}=34)$.

The sOSCAR level was $320(245,390) \mathrm{pg} / \mathrm{ml}$ in the UA group, $270(187,355) \mathrm{pg} / \mathrm{ml}$ in the USTEMI group and $250(110,325) \mathrm{pg} / \mathrm{ml}$ in the STEMI group, respectively. The sOSCAR level was significantly higher in the UA group than in the STEMI group $(P=0.008)$, but it did not show significant differences between the UA group and USTEMI group $(P=0.279)$ as well as the STEMI group and USTEMI group $(P=0.332)$.

MACE occurred in $0(0 \%, 0 / 29)$ patient of the UA group, in 9 patient $(20 \%, 9 / 45)$ of the USTEMI group and in 8 patients $(23.53 \%, 8 / 34)$ of the STEMI group.

\section{Discussion}

ACS presents clinically as a group of clinical syndromes including the rupture or invasion of coronary atherosclerotic plaques and secondary complete or incomplete occlusive thrombosis. For the ACS, an acute and severe cardiovascular disease, the medical treatment is difficult, its mortality is high, and its clinical manifestation and prognosis are very various, so early judgement for ACS prognosis is very important for the patients with ACS. The human OSCAR, a cell surface molecule, is involved in osteoclast differentiation and bone metabolism ${ }^{6}$. OSCAR may play an important role in vascular inflammation or plaque vulnerability during atherosclerosis ${ }^{7}$. Our previous studies have also confirmed that sOSCAR has an independent correlation with the occurrence of $\mathrm{ACS}^{8}$. In this study, we further explored the relationship between sOSCAR level and ACS prognosis.

Our results indicated that the sOSCAR level was significantly lower in the MACE group than in the nonMACE group, and the area under ROC curve of sOSCAR level for predicting MACE occurring within 30 days after CAS was 0.860 with cut-off of $190 \mathrm{pg} / \mathrm{ml}$, specificity of $76.84 \%$ and sensitivity of $87.50 \%$, suggesting that MACE more easily occurs in the ACS patients with serum sOSCAR $<190 \mathrm{pg} / \mathrm{ml}$. Multivariate Logistic regression analysis indicated that low serum SOSCAR level was an independent risk factor of MACE occurring within 30 days after ACS.

OSCAR is a member of the lymphocyte receptor complex family ${ }^{3}$, and is associated with osteoarticular diseases and osteoporosis ${ }^{9,10}$. Herman et al. ${ }^{11}$ found that the serum sOSCAR level was significantly lower in the patients with rheumatoid arthritis than in the normal individuals, and was negatively correlated with rheumatoid arthritis disease activity, so they believed that sOSCAR might have a protective effect. Goettsch et al. ${ }^{12}$ reported that OSCAR, an immune mediator and osteoclast differentiation regulator, is also involved in inflammatory reaction and cell activation during arteriosclerosis. Sinningen et al. ${ }^{7}$ have described that oxLDL, a atherosclerotic stimulator, can promote OSCAR expression on monocytes, so OSCAR may play an important role in vascular inflammation or plaque vulnerability during atherosclerosis. The oxLDL is from oxidation of low density lipoprotein (LDL), and is closely related to early atherosclerosis, because it can induce foam cell production, activate endothelial cell apoptosis and change gene expression, produce pro-inflammatory and 


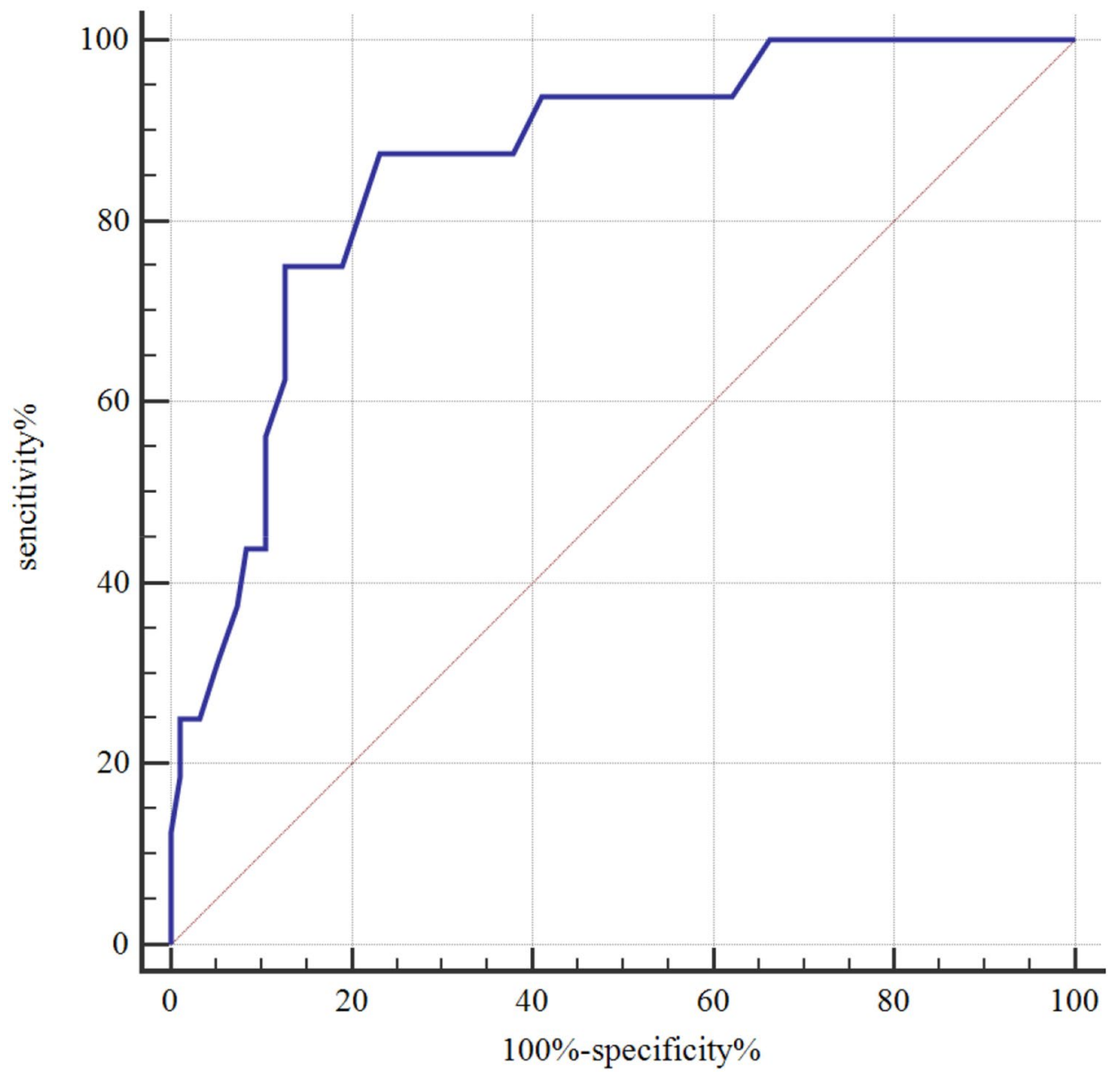

Figure 1. ROC curve of sOSCAR level for predicting MACE. Notes: $R O C$ receiver operating characteristic; sOSCAR soluble osteoclast-associated receptor; MACE major adverse cardiovascular events.

\begin{tabular}{|l|l|l|l|}
\hline Factors & OR values & 95\%CI & P values \\
\hline NT-proBNP & 6.13 & $1.247-30.179$ & 0.01 \\
\hline LVEF & 0.21 & $0.072-0.613$ & 0.03 \\
\hline sOSCAR & 0.26 & $0.087-0.777$ & 0.04 \\
\hline Creatinine & 2.13 & $0.319-14.153$ & 0.25 \\
\hline Age & 2.20 & $0.321-15.071$ & 0.13 \\
\hline
\end{tabular}

Table 2. Independent risk factors of MACE occurring within 30 days after ACS by multivariate Logistic regression analysis. MACE major adverse cardiovascular events; $A C S$ acute coronary syndrome; NT-proBNP $\mathrm{N}$-terminal pro brain natriuretic peptide; $L V E F$ left ventricular ejection fraction; $s O S C A R$ soluble osteoclastassociated receptor.

atherosclerotic stimulation, and regulate costimulatory molecules in endothelial cells ${ }^{13,14}$. OSCAR is expressed in vascular endothelial cells and is regulated by oxLDL, oxLDL regulates OSCAR expression by calcium/nFAT dependent manner, OSCAR promotes the migration of monocytes across endothelial cells and participates in pro-inflammatory cascade reaction ${ }^{3}$. Therefore, OSCAR is involved in atherosclerotic diseases such as ACS probably with oxLDL as a mediator. Our previous studies have also confirmed that low SOSCAR is an independent risk factor of $\mathrm{ACS}^{8}$. This study further found that low sOSCAR level was closely related to MACE in ACS patients, so high serum sOSCAR level might have a protective effect. This is similar to the conclusion of Herman et al. ${ }^{11}$, but the specific protective mechanism remains to be further explored.

Based on our results, the MACE incidence (0\%) was the lowest but the sOSCAR level was the highest in the UA group, while in the STEMI group, the MACE incidence $(23.53 \%)$ was the higest but the sOSCAR level was the lowest among UA, NSTEMI and STEMI groups. Furthermore, the sOSCAR level was significantly higher 
in the UA group than in the STEMI group $(P=0.008)$. Therefore, we can conclude that the higher the sOSCAR level, the lower the MACE incidence.

There are some limitations in this study. Firstly, the sample size was small, and secondly the follow-up time was short. We will expand the sample size and prolong the follow-up time to further confirm the correlation between the serum sOSCAR level and MACE after ACS in future studies.

In summary, serum sOSCAR level is related to ACS prognosis and may be used as a biomarker to predict MACE occurrence after ACS. The higher the sOSCAR level, the lower the MACE incidence.

Received: 16 March 2021; Accepted: 17 May 2021

Published online: 01 June 2021

\section{References}

1. Zhang, W., Chang, G., Cao, L. \& Ding, G. Dysregulation of serum miR-361-5p serves as a biomarker to predict disease onset and short-term prognosis in acute coronary syndrome patients. BMC Cardiovasc. Disord. 21, 74 (2021).

2. Ndongo-Thiam, N., De Sallmard, G., Kastrup, J. \& Miossec, P. Levels of soluble osteoclast-associated receptor (sOSCAR) in rheumatoid arthritis: link to disease severity and cardiovascular risk. Ann. Rheum. Dis. 73, 1276-1277 (2014).

3. Merck, E. et al. Ligation of the FcR gamma chain-associated human osteoclast-associated receptor enhances the proinflammatory responses of human monocytes and neutrophils. J. Immunol. 176, 3149-3156 (2006).

4. O'gara, P. et al. 2013 ACCF/AHA guideline for the management of ST-elevation myocardial infarction: a report of the American College of Cardiology Foundation/American Heart Association Task Force on Practice Guidelines. Circulation 127, 362-425 (2013).

5. Roffi, M. et al. 2015 ESC guidelines for the management of acute coronary syndromes in patients presenting without persistent ST-segment elevation: Task Force for the Management of Acute Coronary Syndromes in Patients Presenting without Persistent ST-Segment Elevation of the European Society of Cardiology (ESC). Eur. Heart J. 37, 267-315 (2016).

6. Han, S. \& Kim, Y. Berberine suppresses RANKL-induced osteoclast differentiation by Inhibiting c-Fos and NFATc1 expression. Am. J. Chin. Med. 47, 439-455 (2019).

7. Sinningen, K. et al. Monocytic expression of osteoclast-associated receptor (OSCAR) is induced in atherosclerotic mice and regulated by oxidized low-density lipoprotein in vitro. Biochem. Biophys. Res. Commun. 437, 314-318 (2013).

8. Zhang, Q. et al. Correlations of soluble osteoclast-associated receptor (sOSCAR) with acute coronary syndrome. Ann. Transl. Med. 6, 408 (2018).

9. Dharmapatni, A. et al. Osteoclast-associated receptor (OSCAR) distribution in the synovial tissues of patients with active RA and TNF- $\alpha$ and RANKL regulation of expression by osteoclasts in vitro. Inflammation 40, 1566-1575 (2017).

10. Crotti, T., Dharmapatni, A., Alias, E., Alias, E. \& Haynes, D. R. Osteoimmunology: major and costimulatory pathway expression associated with chronic inflammatory induced bone loss. J. Immunol. Res. 2015, 281287 (2015).

11. Herman, S. et al. Induction of osteoclast-associated receptor, a key osteoclast costimulation molecule, in rheumatoid arthritis. Arthritis Rheum. 58, 3041-3050 (2008).

12. Goettsch, C. et al. The osteoclast-associated receptor (OSCAR) is a novel receptor regulated by oxidized low-density lipoprotein in human endothelial cells. Endocrinology 152, 4915-4926 (2011).

13. Lee, J. et al. Fluid shear stress regulates the expression of Lectin-like oxidized low density lipoprotein receptor-1 via KLF2-AP-1 pathway depending on its intensity and pattern in endothelial cells. Atherosclerosis 270, 76-88 (2018).

14. Zhang, H. et al. Recombinant human thioredoxin-1 protects macrophages from oxidized low-density lipoprotein-induced foam cell formation and cell apoptosis. Biomol. Ther. 26, 121-129 (2018).

\section{Acknowledgements}

This study was supported by 2019 Nantong City Science and Technology Plan (Guidance) Project (No. MSZ19149), Nantong University School-level Fund Project (No. 2019LZ004) and Jiangsu Province "Six Great Talent Peak" High Level Talent Project (2019-WSW-199).

\section{Author contributions}

R.W. and J.W. wrote the main manuscript text, L.X. and H.C. prepared Figs. 1, Y.Z. and Q.Z. prepared Tables 1-2.

\section{Competing interests}

The authors declare no competing interests.

\section{Additional information}

Correspondence and requests for materials should be addressed to Q.Z.

Reprints and permissions information is available at www.nature.com/reprints.

Publisher's note Springer Nature remains neutral with regard to jurisdictional claims in published maps and institutional affiliations.

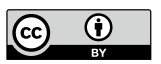

Open Access This article is licensed under a Creative Commons Attribution 4.0 International License, which permits use, sharing, adaptation, distribution and reproduction in any medium or format, as long as you give appropriate credit to the original author(s) and the source, provide a link to the Creative Commons licence, and indicate if changes were made. The images or other third party material in this article are included in the article's Creative Commons licence, unless indicated otherwise in a credit line to the material. If material is not included in the article's Creative Commons licence and your intended use is not permitted by statutory regulation or exceeds the permitted use, you will need to obtain permission directly from the copyright holder. To view a copy of this licence, visit http://creativecommons.org/licenses/by/4.0/.

(C) The Author(s) 2021 